\title{
Improving radiologists' and orthopedists' QoE in diagnosing lumbar disk herniation using 3D modeling
}

\author{
Sanaa Abu Alasal ${ }^{1}$, Mohammad Alsmirat ${ }^{2}$, Asma'a Al-Mnayyis ${ }^{3}$, Qanita Bani baker ${ }^{4}$, Mahmoud \\ Al-Ayyoub ${ }^{5}$ \\ 1,2,4,5 Department of Computer Science, Jordan University of Science and Technology, Jordan \\ ${ }^{2}$ Department of Computer Science, University of Sharjah, United Arab Emirates \\ ${ }^{3}$ Department of Clinical Sciences-Radiology Division-MSK radiologist, Faculty of Medicine, Yarmouk University, Jordan
}

\begin{tabular}{|c|c|}
\hline Article Info & ABSTRACT \\
\hline Article history: & \multirow{10}{*}{$\begin{array}{l}\text { This article studies and analyzes the use of } 3 \mathrm{D} \text { models, built from magnetic reso- } \\
\text { nance imaging (MRI) axial scans of the lumbar intervertebral disk, that are needed } \\
\text { for the diagnosis of disk herniation. We study the possibility of assisting radiologists } \\
\text { and orthopedists and increasing their quality of experience (QoE) during the diagno- } \\
\text { sis process. The main aim is to build a 3D model for the desired area of interest and } \\
\text { ask the specialists to consider the 3D models in the diagnosis process instead of con- } \\
\text { sidering multiple axial MRI scans. We further propose an automated framework to } \\
\text { diagnose the lumber disk herniation using the constructed 3D models. We evaluate the } \\
\text { effectiveness of increasing the specialists QoE by conducting a questionnaire on } 14 \\
\text { specialists with different experiences ranging from residents to consultants. We then } \\
\text { evaluate the effectiveness of the automated diagnosis framework by training it with a } \\
\text { set of } 83 \text { cases and then testing it on an unseen test set. The results show that the the } \\
\text { use of 3D models increases doctors QoE and the automated framework gets } 90 \% \text { of } \\
\text { diagnosis accuracy. }\end{array}$} \\
\hline Received Oct 29, 2020 & \\
\hline Revised Mar 4, 2021 & \\
\hline Accepted Mar 5, 2021 & \\
\hline Keywords: & \\
\hline 3D modeling & \\
\hline Disk herniation & \\
\hline Isosurface & \\
\hline Marching cubes & \\
\hline MRI & \\
\hline
\end{tabular}

This is an open access article under the CC BY-SA license.

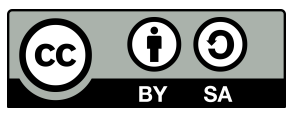

\section{Corresponding Author:}

Mohammad Alsmirat

University of Sharjah and Jordan University of Science and Technology

United Arab Emirates

Email: malsmirat@sharjah.ac.ae,masmirat@just.edu.jo

\section{INTRODUCTION}

A human spine consists of a set of bones, each is called vertebrae, that are separated by a filler called a disk. A disk degradation, usually called disk herniation, is a local displacement of disk ingredients toward the posterior normal margins of the intervertebral disk [1]. Lumbar intervertebral disk herniation (IDH) is a disease that causes lower back pain (LBP). The traditional way of diagnosing this disease is by inspecting 2D radiological images, such as magnetic resonance imaging (MRI) scans. The results of this manual eye analysis might be inaccurate due to the differences in diagnosis from a radiologist/orthopedist to another, which is known as intra- and inter-radiologist variation. Moreover, bad quality of MRI scans in some cases may lead to an incorrect decision on a patient case. Besides, training new specialists to read MRI scans and diagnose cases requires senior specialists to devote their valuable time for the process. To deal with the aforementioned challenges, computer aided systems (CAD) can be utilized to assist the specialists to work and gain experience [2].

MRI is currently the best way for visualizing the spine and for evaluating IDH. Providing a high soft tissue contrast, it allows good visualization of the intervertebral disk in addition to the surrounding nerves, 
ligaments, and muscles. This makes MRI a routine and a key modality in managing and diagnosing such diseases [2]. It is usually used to diagnose patients suffering from spinal stenosis, disk herniation, or tumors [3]. MRI uses magnetic fields to obtain a set of multi-planar images of the body organs. Those anatomic details of the 3D organ are presented as a set of 2D parallel cross-sectional axial, sagittal and coronal images [4].

We can find many researches in the literature that deals with automatic diagnosis based on clinical MRI data, but they have mostly used only the sagittal views [1], [3] [5]-[11]. Some other researches focus on converting medical images into quantitative measurements to solve the problem of having to read medical images, which many specialists consider a non-trivial task [3], [12]-[14]. As a new direction, researchers are studying the possibility of building 3D models from medical images of different human organs [10], [11], [14][18] to enhance the diagnosing strategies, which in turns make reading the images easier for the specialists but none of the works dealt with intervertebral disks from axial scans before.

In a recent work [19], we have successfully built a 3D model for lumbar intervertebral disks from a limited number of axial MRI images. The main aim of that study is to build a 3D model from a very limited number of axial MRI slices, as it is very hard to get more slices for that area. Moreover, we used the resulting 3D model in visualizing the disk to assist the specialist in the manual diagnoses. In this paper, we expand this study by surveying the opinion of 14 radiologists and orthopedists with different levels of experience on using the 3D built-in [19] instead of using the MRI axial 2D scans. We also build an automated system that diagnoses the IDH automatically based on the 3D model.

We evaluate the effectiveness of increasing the specialists quality of experience using the image set collected in [19]-[22]. The specialists find it easier to make a diagnosis based only on the 3D model in $95 \%$ of the cases. Moreover, we evaluate the auto-diagnosis system by training it on the same image set and testing it on unseen cases. The system gets the correct diagnosis in $90 \%$ of tested cases.

The rest of this paper is organized as follows. In the next section 2, we discuss our methodology including the dataset we use in our experiments, the proposed 3D model reconstruction, the auto-diagnosing process, and the quality of experience evaluation. Finally, the results are presented in section 3 and the paper is concluded in section 4 .

\section{PROPOSED METHODS}

In this work, we use the same dataset used in our recent work [19], which consists of axial T2 type MRI scans with only three scans taken per each disk (top, middle, and bottom). The area to be diagnosed in each slice can be represented by a line that lies between the posterior of the lumbar disk and the interior of the thecal sac. In a healthy axial disk, disk area should look like a disk of black middle and white margins and the thecal sac area should look like a triangle with openings that the spinal nerves passes through them. These openings are called lateral neural foramen (NF) [23]. The anatomy of lumber disk and its peripheral is provided in our previous work [19]. In the case of disk herniation, the disk appears bulged out the NF fiber that reaches the posterior margin, which touches the thecal sac region. This bulging appears as a black region that spreads from the posterior side to the anterior side of the thecal sac and NF. A radiologist examined these cases and provided ground truth for this $2 \mathrm{D}$ dataset. The dataset contains 83 cases divided into 45 abnormal cases and 38 normal cases. For the abrormal cases, 30 cases are found to be diffuse, one found to be left-foraminal, one found to be right-foraminal and 13 cases found to be bi-foraminal.

In this research, the main objective is to diagnose the lumber disk herniation from the 3D mesh generated by visualizing the MRI slices as a stack. The 3D modeling system we use is the one proposed and described in details in [19] which passes through the following stages:

- Region of interest (ROI) extraction from the MRI 2D scans using the heuristic technique described in [20]-[22].

- ROI enhancement by preprocessing the extracted ROI to emphasize the edges that separate the tissues from each other.

- Filling the gaps between the 2D slices using Interpolation. This step is essential as only three 2D slices are available for each disk.

- 3D model construction and visualization. In this step, the interpolated slices are combined in a stack (3D array). Then, the next step is to extract the isosurfaces and isocaps form the 3D array to visualize the data [24]. This can be done using the marching cubes algorithm, which returns a surface represented by 
a set of triangles (isosurfaces) and a set of end-caps for open ended isosurfaces (isocaps).

- 3D mesh generation. In this step, the isosurface and isocaps are combined in one complete triangulated mesh called Face-Vertex Mesh that is represented using set of vertices and edges.

\subsection{Auto-diagnosing system}

As the area of interest needed to diagnose the cases is the margin between the posterior and the interior side of the thecal sac (the edge between the disk and the thecal sac), we need to segment the surface from the mesh that represents the needed edge. As a starting step, we cut the top $25 \%$ of the mesh resulting from the last step in the previous procedure and continue working on it. This part is shown in Figure 1(a).

The information describing the output mesh from the last step are mesh connectivity and mesh geometry. The mesh connectivity (also known as the topology) describes the incidence relations among mesh elements (such as adjacent vertices and edges of a face). The mesh geometry specifies the position and other geometric characteristics of each vertex.

Considering that the mesh is a graph, then the connectivity of this mesh also follows the graph theory concepts [25]. Therefore, in order to segment the area of interest in the mesh that represents our model, we have to split it into sub-graphs, where each sub-graph is a connected component [25]. Connected components are sub-graphs in which any two vertices in one component are connected to each other by a path, and are not connected to vertices in other components [25], [26]. In this case, the area of interest is the top surface of the mesh.

The segmentation process is performed using either breadth-first search (BFS) or depth-first search (DFS). In either case, the search starts at a particular vertex $u$ and finds the entire connected component that contains $u$. To discover all of the graph's connected components, the algorithm loops through the vertices, until it finds a vertex $v$ that is not already included in a previously found connected component. Then, the search step is repeated to find the component that includes $v$. This process is repeated until the algorithm can no longer find a vertex that does not belong to a component [25]. In our case, the 3D mesh segmentation is done through passing over the isoface sets and counting their vertices. At each time no more vertices are reached, it is assumed that an edge of a separate component is reached. It has been noticed that the largest connected component that can be found in the mesh represents our area of interest. In the automated diagnosis system, this area is extracted and the following analysis in the framework is based on it. An example of a final component is illustrated in Figure 1(b).

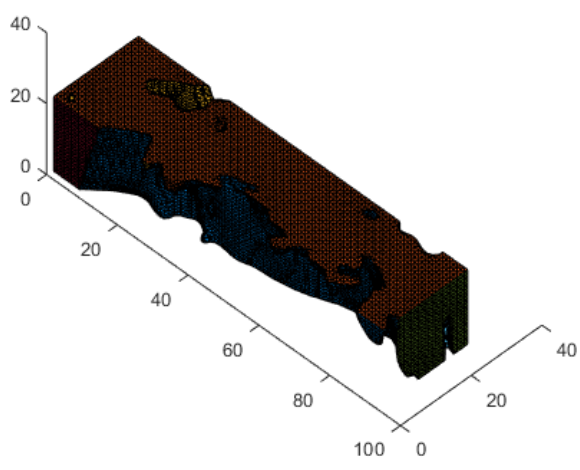

(a)

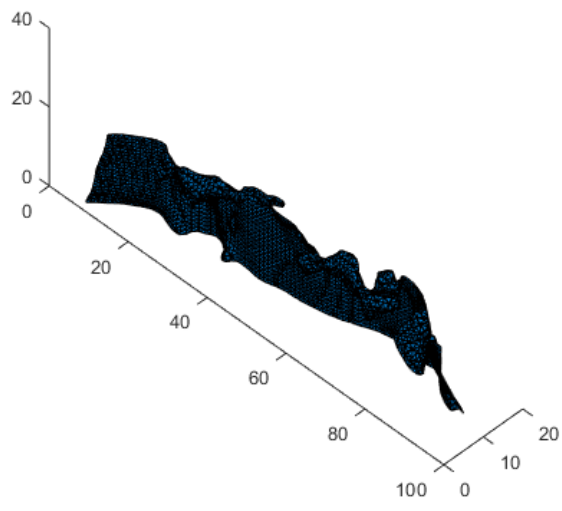

(b)

Figure 1. 3D model segmentation; (a) Segmented mesh, (b) Largest connected component

Given that diagnosing disk herniation cases is usually based on noticing that there is an overlapping between the bulged tear and the thecal sac or the neural foramina, we find that the auto-diagnosing of such a case can be done by finding a corrosion in the model the we have built. If a corrosion (or tear) exists only at the left side of the model, the case represented by the model can be diagnosed as left-foramina. If a corrosion exists at the right side, the case can be diagnosed as right-foramina. Finally, if the corrosion exists in both sides, the case can be diagnosed as bi-foramina. Figure 2 illustrates examples of normal and abnormal cases with corrosion shown in the abnormal case. 


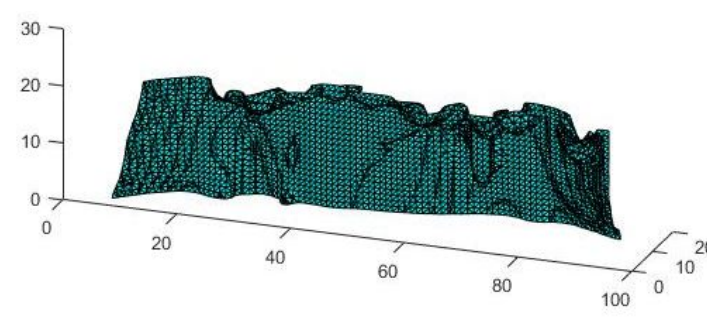

(a)

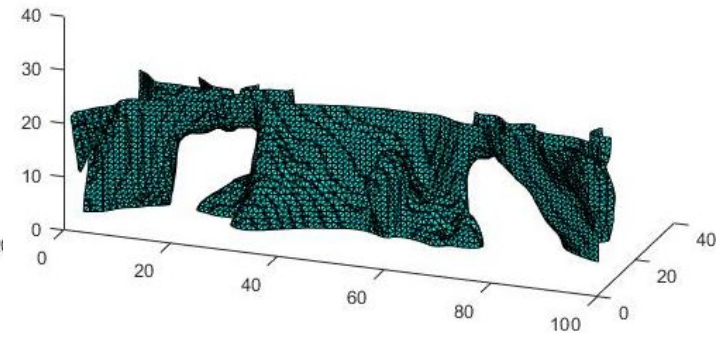

(b)

Figure 2. Examples of; (a) Not corroded mesh, and (b) Corroded one

In order to detect these corrosion areas, we have developed a method to cut the 3D model mesh from the middle horizontally in the $Z$ axis to get two pieces and extract the number of connected components in each part. If the number of components is more than one in one part, this mean that the part has corrosion and this mean that the case is abnormal. Otherwise, the case is diagnosed to be normal. If the case is abnormal and we get only two components, one of them is smaller than the other; If the smaller component is located to the right, the case is diagnosed to be right-foramina. On the other hand, if the smaller component is located to the left, the case is diagnosed as left-foramina. If we have three components or more, this case called bi-foramina. To perform the mesh cutting, the developed method looks for the vertices close to the position of the cut from the vertices structure and extracts the needed faces from the faces structure. After that, new structures are created to store the vertices and the faces for each part. The process should make sure not to duplicate vertices in different structures.

\subsection{Quality of experience evaluation}

To measure the usefulness of using the 3D models by specialists in the manual diagnosis process and its impact of their quality of experience (QoE), we have conducted a questionnaire (scored using 5-point Likert scale from strongly agree (score 5) to strongly disagree (score 1)). The targeted groups during the assessment process are orthopedic surgeons, radiologists, and general surgeons, at different levels of experience ranging from resident to specialist and even consultant. Moreover, to make the use of the 3D model easier for the target group, we have developed an application with a graphical user interface (GUI). Through this GUI, the user can browse for the files of the case he/she wants to check. Then, he/she can run seven different functions button. The system GUI is shown in Figure 3 and a sample run that shows the result of each function is shown in Figure 4.

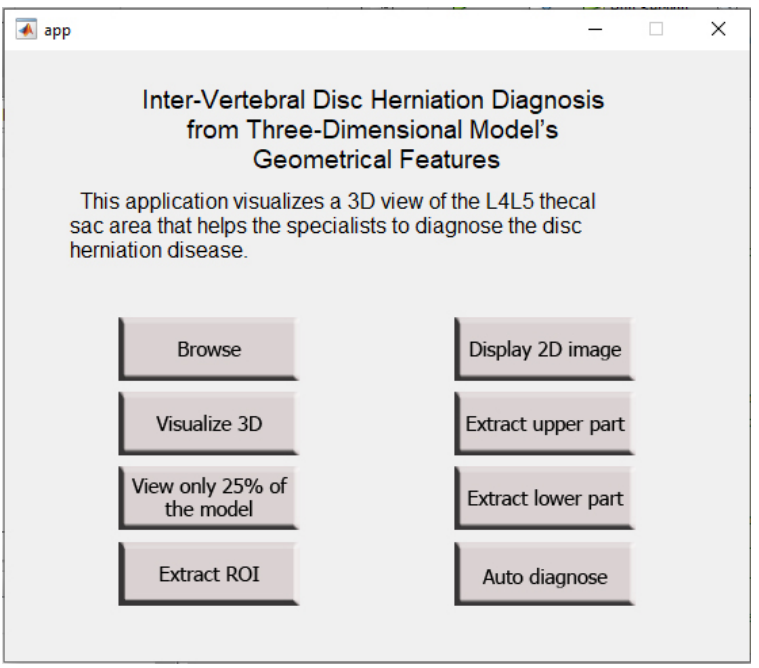

Figure 3. Application interface 


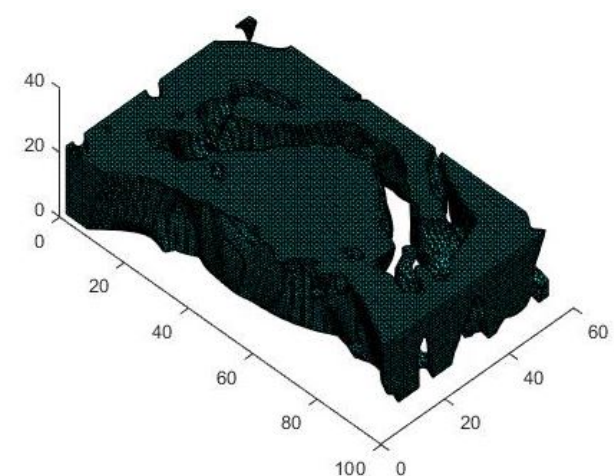

(a)

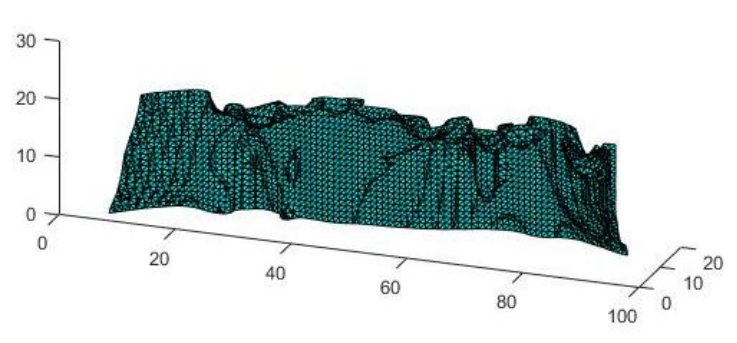

(c)

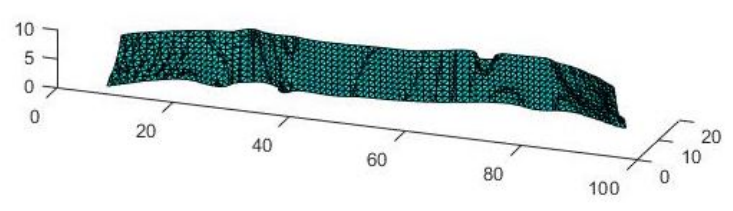

(e)

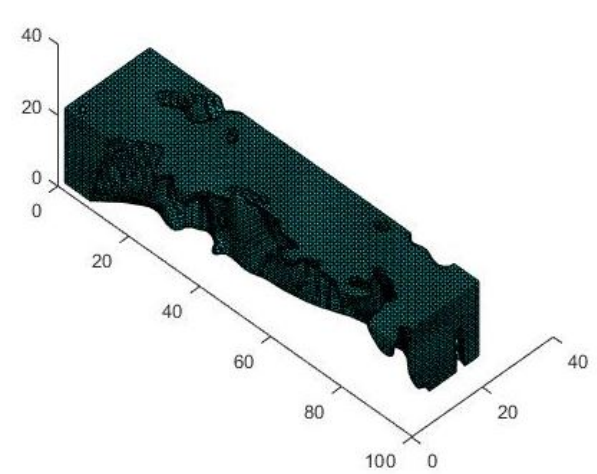

(b)

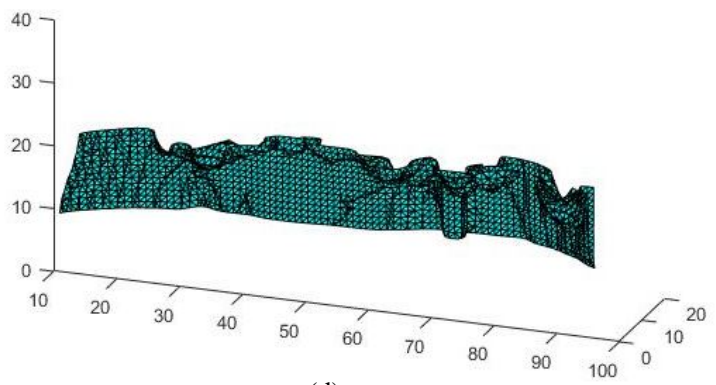

(d)

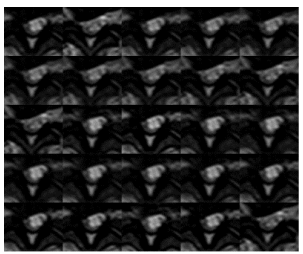

(f)

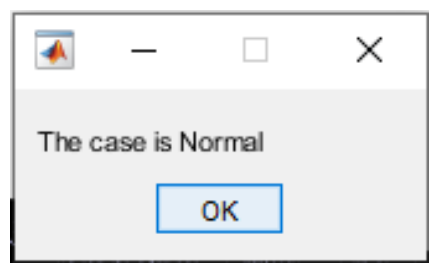

(g)

Figure 4. Run Example; (a) Visualize 3D, (b) View only 25\% of the model, (c) Extract ROI, (d) Extract upper part, (e) Extract lower part, (f) View 2D images, and (g) Auto diagnose

The questionnaire tests the system from different points of view such as usability, clarity, dependability, and confidence for making decisions in the diagnosing process. The questionnaire also tests whether or not the specialists can rely on the system in a way that removes the need to open 2D views of MRI scans (which is the common practice), and whether the system can replace the radiologist or not. The questions are shown in Table 1.

The survey participants included 14 users. Their distribution is shown in Table 2. We have noted that the idea of using CAD systems is more acceptable among specialists and residents, while it was difficult to even discuss the idea with consultants. This resistance to change can be explained by the fact that consultants believe that their expertise is sufficient in diagnoses, despite the fact that some studies (such as the one in [2]) show that $2 \mathrm{D}$ images do not give sufficient information about the dissection of the imaging area even if they are 
MRIs. The statistical analysis was performed using IBM SPSS Statistics 25, so that we can study the usability and acceptance of our work by physicians who directly deal with the application.

Table 1. QoE Questionnaire

\begin{tabular}{|c|c|c|c|c|c|c|}
\hline \multicolumn{7}{|c|}{ Strongly agree (5), Agree (4), Neutral (3), Dis-agree (2), Strongly disagree (1) } \\
\hline & Question & 5 & 4 & 3 & 2 & 1 \\
\hline 1 & Was the system easy to use for you? & & & & & \\
\hline 2 & Did you need the assistant of the researcher regarding how to use the system? & & & & & \\
\hline 3 & Is it easy to understand the 3D model shape and appearance? & & & & & \\
\hline 4 & Is the $3 \mathrm{D}$ model representative enough to preform the diagnosing strategies? & & & & & \\
\hline 5 & Was the area of interest clear for all cases? & & & & & \\
\hline 6 & Was the diagnosing process easy? & & & & & \\
\hline 7 & Did you need the 2D images during the diagnosing process? & & & & & \\
\hline 8 & Do you recommend such a system to assist in the diagnosing process? & & & & & \\
\hline 9 & Do you expect that such systems will replace the radiologist in the future? & & & & & \\
\hline 10 & How confident are you with such systems? & & & & & \\
\hline 11 & Do you prefer 3D Systems over 2D Systems? & & & & & \\
\hline
\end{tabular}

Table 2. Distribution of survey participants

\begin{tabular}{lllll}
\hline & & \multicolumn{3}{c}{ Specialization } \\
& & Orthopedic & Radiologist & General Surgery \\
\hline 3*Level & Consultant & 2 & 1 & 0 \\
& Specialist & 5 & 1 & 0 \\
& Resident & 3 & 0 & 2 \\
\hline
\end{tabular}

\section{RESULTS AND DISCUSSION}

\subsection{D modeling validation results}

We have to validate the resulting models and a ground truth is needed for this purpose. Unfortunately, it is impossible to have a ground truth 3D model that models lumbar disks as they may differ from one person to another. For that reason, with the help of a radiologist, the diagnosis of the cases in our dataset is performed by visualizing only the $3 \mathrm{D}$ models rather than analyzing the set of $2 \mathrm{D}$ slices. Our $3 \mathrm{D}$ modeling framework is validated by comparing the $2 \mathrm{D}$ diagnosis results versus the $3 \mathrm{D}$ diagnosis results.

There were only four cases in which the radiologist needed to get back to the 2D images. After investigating these cases, we find that the problem in the construction of the model is caused by some erroneous slices that may result from partial volume overlap between the disk region and the bone of the upper or lower vertebrae.

To evaluate the 3D models, we use the accuracy as an evaluation metric, which is typical in systems like ours. To explain what this measure means, we have to define the following terminology. The TP and FP are the numbers of true positive cases and false positive cases, respectively. A case is true positive if it is a normal case that is correctly classified as normal by our system, whereas a false positive case is an abnormal case that is incorrectly classified as normal by our system. On the other hand, TN and FN are the numbers of true negative cases and false negative cases, respectively. A true negative case is an abnormal case that is correctly classified as abnormal by the system, and a false negative case is a normal case that is incorrectly classified as abnormal by the system. Thus, we can calculate the accuracy (Ac), which is the percentage of correctly classified cases, as follows:

$$
A c=((T P+T N)) /(P+N)
$$

By quantitatively measuring, the evaluation shows that the proposed 3D model construction has an accuracy of $95 \%$ depending on the values in Table 3, where 36 out of 38 normal cases and 43 out of 45 abnormal cases are properly classified. For the abnormals, one is left-foramina, another one is right-foramina, 13 are bi-foramina and the remaining ones are diffuse.

Table 3. Confusion matrix for the 3D model validation

\begin{tabular}{ccc}
\hline & Normal & Abnormal \\
\hline Normal & 36 & 2 \\
Abnormal & 2 & 43 \\
\hline
\end{tabular}


We also test our auto-diagnosing system by applying it on unseen data. We obtain $90 \%$ accuracy compared with the $2 \mathrm{D}$ ground truth built by a radiologist. The unseen cases contain five normal cases and five abnormal (three bi-foramina, one left-foramina, and one right-foramina). The confusion matrix is presented in Table 4.

Table 4. Confusion matrix for the auto diagnosing system validation

\begin{tabular}{ccc}
\hline & Normal & Abnormal \\
\hline Normal & 5 & 0 \\
Abnormal & 1 & 4 \\
\hline
\end{tabular}

\subsection{Survey results}

The doctors" opinions were divided between "strongly agree" and "agree" with respect to the confidence in such a system. The question was: Can a computer system be used to diagnose a patient's condition?. The answer was that if all the information were available and analyzed correctly, it would be possible to rely on these systems. An illustration is provided by Table 5 .

Table 5. How much confidence in the system does the participants have?

\begin{tabular}{|c|c|c|c|c|c|c|}
\hline \multicolumn{7}{|c|}{ Confidence } \\
\hline Level & Specialization & Strongly disagree & Disagree & Neutral & Agree & Strongly agree \\
\hline \multirow[t]{3}{*}{$3 *$ Consultant } & Orthopedic & 0 & 0 & 0 & 1 & 1 \\
\hline & Radiologist & 0 & 0 & 0 & 0 & 1 \\
\hline & General surgery & 0 & 0 & 0 & 0 & 0 \\
\hline \multirow[t]{3}{*}{$3 *$ Specialist } & Orthopedic & 0 & 0 & 0 & 5 & 0 \\
\hline & Radiologist & 0 & 0 & 0 & 0 & 0 \\
\hline & General surgery & 0 & 0 & 0 & 0 & 0 \\
\hline \multirow[t]{3}{*}{$3 *$ Resident } & Orthopedic & 0 & 0 & 1 & 0 & 2 \\
\hline & Radiologist & 0 & 0 & 0 & 0 & 0 \\
\hline & General surgery & 0 & 0 & 0 & 1 & 1 \\
\hline
\end{tabular}

Table 6 shows the relationship between the ease of diagnosis and the doctor's specialty and level. The table shows that it is more acceptable for orthopedics specialists to diagnose through our system. For the radiologist's replacement question, the vast majority of doctors had objected. Therefore, depending on the study of the previous factors, we conclude that the 3D system is satisfactory in the case of assisting doctors in the diagnosis of those specific cases that are difficult to diagnose from the 2D images, such as the presence of inconvenience or the far-distance between slices. However, it cannot replace one of the major doctors' jobs. Table 7 illustrates the results.

Regarding the 2D image opening factor, it has been shown that it can be dispensed with by specialists and residents of different specialties, where ten doctors voted in agreement, while the remaining four were distributed between neutral and disagreement. Table 8 shows the analysis of the 2D images opening. Finally, we have satisfactory level of acceptance by the survey participants distributed across different specialization. The best reactions were by the specialists and residents, while there was a kind of rejection from some consultants in terms of reliance on the automated system of diagnosis; with the satisfaction of the existence of such 3D system that supports the diagnosing. And hence, we predict that 3D decision support methods might be used in the educational process in the near future.

Table 6. How easy was the diagnosing process for the participants?

\begin{tabular}{lllllll}
\hline \multirow{2}{*}{ Level } & \multicolumn{3}{c}{ Easly diagnosing } & & & \\
& Specialization & Strongly disagree & Disagree & Neutral & Agree & Strongly agree \\
\hline \multirow{3}{*}{ 3* Spensultant } & Orthopedic & 0 & 0 & 0 & 1 & 1 \\
& Radiologist & 0 & 0 & 0 & 1 & 0 \\
& General surgery & 0 & 0 & 0 & 0 & 0 \\
$3 *$ Resident & Orthopedic & 0 & 0 & 0 & 1 & 4 \\
& Radiologist & 0 & 0 & 0 & 0 & 1 \\
& General surgery & 0 & 0 & 0 & 0 & 0 \\
& Orthopedic & 0 & 0 & 0 & 1 & 2 \\
& Radiologist & 0 & 0 & 0 & 0 & 0 \\
& General surgery & 0 & 0 & 0 & 1 & 1 \\
\hline
\end{tabular}


Table 7. The participants' acceptance of idea that this system can replace the radiologists

\begin{tabular}{lllllll}
\hline \multirow{2}{*}{ Level } & Specialization & Strongly disagree & Disagree & Neutral & Agree & Strongly agree \\
\hline \multirow{3}{*}{ 3*Consultant } & Orthopedic & 0 & 1 & 1 & 0 & 0 \\
& Radiologist & 1 & 0 & 0 & 0 & 0 \\
& General surgery & 0 & 0 & 0 & 0 & 0 \\
$3 *$ Specialist & Orthopedic & 1 & 2 & 2 & 0 & 0 \\
& Radiologist & 0 & 0 & 1 & 0 & 0 \\
& General surgery & 0 & 0 & 0 & 0 & 0 \\
& Orthopedic & 0 & 1 & 0 & 0 & 2 \\
& Radiologist & 0 & 0 & 0 & 0 & 0 \\
& General surgery & 1 & 0 & 1 & 0 & 0 \\
\hline
\end{tabular}

Table 8 . The need to open the original 2D images

\begin{tabular}{lllllll}
\hline \multirow{2}{*}{ Level } & \multicolumn{3}{c}{ Need to open 2D images } & & & \\
& Specialization & Strongly disagree & Disagree & Neutral & Agree & Strongly agree \\
\hline \multirow{3}{*}{$3 *$ Consultant } & Orthopedic & 0 & 0 & 0 & 1 & 1 \\
& Radiologist & 0 & 1 & 0 & 0 & 0 \\
& General surgery & 0 & 0 & 0 & 0 & 0 \\
$3 *$ Residentist & Orthopedic & 1 & 1 & 0 & 2 & 1 \\
& Radiologist & 0 & 0 & 1 & 0 & 0 \\
& General surgery & 0 & 0 & 0 & 0 & 0 \\
& Orthopedic & 0 & 0 & 0 & 1 & 2 \\
& Radiologist & 0 & 0 & 0 & 0 & 0 \\
& General surgery & 0 & 0 & 0 & 0 & 2 \\
\hline
\end{tabular}

\section{CONCLUSION}

In this paper, we presented a simple, robust, and fully automated image analysis framework for spinal images with a special focus on axial MRI of the lumbar spine. In addition, our framework was tested on a dataset that contains 83 patient cases. We first extracted the ROI to be as the radiologist recommended. The next step was to enhance the ROI edges, by sharpening a copy of the image and adding it again to the original image. The ROI is then interpolated the slices to fill the gaps between the slides, as these distances may change the texture of the disk slices. Then, we stacked the images in a 3D matrix to be used in the extraction of the model that will be analyzed later. This extraction expresses keeping the intensity value of the tissue needed in the diagnostic method. The value was selected upon some test for more than isovalue, as it gave the desired shape and details of the area. At the end, the accuracy measurements and survey results showed satisfaction regarding our work. We concluded that the 3D modeling has great impact on the visualizing of the patient cases in addition to enhancing the QoE of specialists leading to great supplement to the diagnosing process.

\section{REFERENCES}

[1] A. H. Mir, "Segmentation of the herniated intervertebral discs," International Journal of Image, Graphics and Signal Processing, vol. 10, no. 6, pp. 31-41, 2018, doi: 10.5815/ijigsp.2018.06.04.

[2] S. Ghosh, V Chaudhary, and G. Dhillon, "Exploring the utility of axial lumbar mri for automatic diagnosis of intervertebral disc abnormalities," Medical Imaging 2013: Computer-Aided Diagnosis, vol. 8670, 2013, Art. No. 86703D-2, doi: 10.1117/12.2007704.

[3] Meelis Lootus, "Automated radiological analysis of spinal MRI," PhD thesis, University of Oxford, 2015.

[4] N. Archip, R. Rohling, V. Dessenne, P.-J. Erard, and L. P. Nolte, "Anatomical structure modeling from medical images," Computer Methods and Programs in Biomedicine, vol. 82, no. 3, pp. 203-215, 2006, doi: 10.1016/j.cmpb.2006.04.009.

[5] A. Raja'S, J. J. Corso, and V. Chaudhary, "Labeling of lumbar discs using both pixel-and object-level features with a two-level probabilistic model," IEEE transactions on medical imaging, vol. 30, no. 1, pp. 1-10, 2011, doi: 10.1109/TMI.2010.2047403.

[6] S. Michopoulou, "Image analysis for the diagnosis of MR images of the lumbar spine," PhD thesis, UCL (University College London), 2011.

[7] A. Raja'S, V. Chaudhary, and G. Dhillon, "Computer aided diagnosis system for lumbar spine," in Proceedings of the 4th International Symposium on Applied Sciences in Biomedical and Communication Technologies, 2011, pp. 1-5, doi: 10.1145/2093698.2093843. 
[8] A. Raja'S, J. J. Corso, V. Chaudhary, and G. Dhillon, "Automatic diagnosis of lumbar disc herniation with shape and appearance features from MRI," In Medical Imaging 2010: Computer-Aided Diagnosis, vol. 7624, 2010, Art. No. 76241A, doi: 10.1117/12.842199.

[9] A. Raja'S, J. J. Corso, V. Chaudhary, and G. Dhillon, “Toward a clinical lumbar cad: herniation diagnosis," International journal of computer assisted radiology and surgery, vol. 6, no. 1, pp. 119-126, 2011, doi: 10.1007/s11548-0100487-7.

[10] R. Haq, R. Aras, D. A. Besachio, and R. C. Borgie, and M. A Audette, "3d lumbar spine intervertebral disc segmentation and compression simulation from mri using shape-aware models," International journal of computer assisted radiology and surgery, vol. 10, no. 1, pp. 45-54, 2015, doi: 10.1007/s11548-014-1094-9.

[11] J. B. Mendieta, "An efficient and semiautomatic segmentation method for 3D surface reconstruction of the lumbar spine from Magnetic Resonance Imaging (MRI),” PhD thesis, Queensland University of Technology, 2016.

[12] A. Beulah, T. Sree Sharmila, and V. K. Pramod, "Disc bulge diagnostic model in axial lumbar mr images using intervertebral disc descriptor (idd)," Multimedia Tools and Applications, vol. 77, no. 20, pp. 27215-27230, 2018, doi: 10.1007/s11042-018-5914-8.

[13] J. Yao, T. Klinder, and S. Li, "Computational Methods and Clinical Applications for Spine Imaging," Springer, 2014.

[14] S. M. Ali, L. K. Abood, R. S. Abdoon, "Automatic technique to produce 3d image for brain tumor of mri images," Journal of University of Babylon, vol. 22, no. 7, pp. 1896-1907, 2014,

[15] M. P. Arakeri, G. R. M. Reddy, R. Mohana, "An effective and efficient approach to 3d reconstruction and quantification of brain tumor on magnetic resonance images," International Journal of Signal Processing, Image Processing and Pattern Recognition, vol. 6, no. 3, pp. 111-128, 2013.

[16] C. Kumar, A. Kumari, "3d reconstruction of brain tumor from 2d MRI's using fcm and marching cubes," International Journal of Advanced Research in Electronics and Communication Engineering, vol. 3, no. 9, pp. 970-974, 2014.

[17] C. P Loizou, C. Papacharalambous, G. Samaras, E. Kyriacou, T. Kasparis, and M. Pantziaris et al., "Brain image and lesions registration and 3d reconstruction in dicom MRI images," In 2017 IEEE 30th International Symposium onComputer-Based Medical Systems (CBMS), 2017, pp. 419-422, doi: 10.1109/CBMS.2017.53.

[18] H. Bashir, F. Hussain, and M. H. Yousaf, "Smart algorithm for 3d reconstruction and segmentation of brain tumor from mris using slice selection mechanism," Smart Computing Review, vol. 5, no. 3, pp. 187-200, 2015, doi: 10.6029/smartcr.2015.03.007.

[19] A. Al-Mnayyis, S. A. Alasal, M. Alsmirat, Q. B. Baker, and S. AlZu'bi, “ Lumbar disk 3d modeling from limited number of mri axial slices," International Journal of Electrical and Computer Engineering (IJECE), vol. 10, no. 4, pp. 4101-4108, 2020, doi: 10.11591/ijece.v10i4.pp4101-4108.

[20] K. Alawneh, M. Al-dwiekat, M. Alsmirat, and M. Al-Ayyoub, "Computer-aided diagnosis of lumbar disc herniation," In 2015 6th International Conference on Information and Communication Systems (ICICS), 2015, pp. 286-291, doi: 10.1109/IACS.2015.7103190.

[21] M. Al-Ayyoub, N. Al-Mnayyis, M. A Alsmirat, K. Alawneh, Y. Jararweh, and B. B. Gupta, "Sift based roi extraction for lumbar disk herniation cad system from mri axial scans," Journal of Ambient Intelligence and Humanized Computing, pp. 1-9, 2018, doi: 10.1007/s12652-018-0750-2.

[22] M. Alsmirat, K. Alawneh, M. Al-Ayyoub, and M. Al-dwiekat, "Building a simulated educational environment for the diagnosis of lumbar disk herniation using axial view mri scans," Int J Adv Intell Paradig (IJAIP), 2017.

[23] S. Al-Helo, R. S. Alomari, S. Ghosh, V. Chaudhary, G. Dhillon, Al-Zoubi Mohd B et al., "Compression fracture diagnosis in lumbar: A clinical cad system," International journal of computer assisted radiology and surgery, vol. 8, no. 3, pp. 461-469, 2013.

[24] W. J. Schroeder, K. M. Martin, and W. E. Lorensen, "The visualization toolkit: an object-oriented approach to 3D graphics," Kitware, 2006.

[25] J. Kleinberg and E. Tardos, “Algorithm design,” Pearson Education India, 2006.

[26] J. Hopcroft and R. Tarjan, "Algorithm 447: efficient algorithms for graph manipulation," Communications of the ACM, vol. 16, no. 6, pp. 372-378, 1973, doi: 10.1145/362248.362272. 